\title{
A comprehensive literature review of theories and constructs utilized in type 2 diabetes self-care management research involving African-American
}

\section{men}

Ledric D. Sherman

Department of Health and Kinesiology, Division of Health Education, College of Education and Human Development, Texas AEM University, TX, USA

\section{A B S T R A C T}

Introduction: The purpose of this study is to ascertain the extent and quality of theory and theoretical construct utilization among published empirical studies specific to self-care management research involving African-American men living with type 2 diabetes. Materials and Methods: Systematic literature review for peer-reviewed articles was performed. Articles with barriers, facilitators, masculinity, social support, mistrust, behaviors and decision making among this specific group were sought out. Results: Some studies mentioned a specific theoretical framework or model. However, 35 out of fifty studies did not report any use of theoretical framework or model. Conclusions: This paucity of theory utilization points to a breach in the quality of both qualitative and quantitative research in this area with is particular population of men. Researchers either failed to sufficiently use theory or did so but failed to specify how.

Key words: African-American men, constructs, theory, type 2 diabetes

\section{INTRODUCTION}

Diabetes mellitus is an extensive health complication that affects all racial and ethnic groups in the United States. Yet diabetes disproportionately affects AfricanAmerican men and contributes to other leading causes of death such as heart diseases, cancer, unintentional injuries, strokes, and homicide, and was the sixth leading cause of death for African-American men in 2009. ${ }^{[1,2]}$ Type 2 diabetes accounts for $90-95 \%$ of all diabetes cases. ${ }^{[3]}$ Nationally, compared to non-Hispanic Whites, African-Americans have 2-4 times the rates of type 2 diabetes and associated kidney failure, blindness,

\begin{tabular}{|l|l|}
\hline \multicolumn{2}{|c|}{ Access this article online } \\
\hline Quick Response Code: & Website: \\
\hline & www.joshd.net \\
\hline & \\
\hline
\end{tabular}

lower limb amputations, and amputation-related mortalities. ${ }^{[3-5]}$

It is estimated that up to $85 \%$ of lower extremity amputations can be prevented through programs for preventing and treating foot ulcers, preventing reoccurrence of ulcers, and educating patients about proper foot care. ${ }^{[6]}$ Physicians, diabetes researchers, and health educators argue that complications of type 2 diabetes can be prevented because while it is a chronic condition, it is also manageable. Other researchers argue that type 2 diabetes is a complex

This is an open access article distributed under the terms of the Creative Commons Attribution-NonCommercial-ShareAlike 3.0 License, which allows others to remix, tweak, and build upon the work non-commercially, as long as the author is credited and the new creations are licensed under the identical terms.

For reprints contact: reprints@medknow.com

How to cite this article: Sherman LD. A comprehensive literature review of theories and constructs utilized in type 2 diabetes self-care management research involving African-American men. J Soc Health Diabetes 2016;4:127-32. 
disease to manage, especially as most of the care involves self-management. ${ }^{[7]}$ Self-management is defined as the knowledge and skills needed to perform self-care, manage crises, and make the lifestyle changes required to successfully manage a disease. ${ }^{[8]}$

Regardless of severity or complexity, type 2 diabetes requires the patient to monitor and manage their own treatment. The current literature suggests that African-Americans are significantly less adherent to self-management recommendations than non-Hispanic Whites. This may account for the increased complications and mortality rates among this population. ${ }^{[9]}$ Yet, there is little information available to clarify the reasons for the low rates of treatment adherence among AfricanAmericans. Given the disproportionate disease burden and complications among this group, it is important for stakeholders to understand factors which enhance or detract from successful self-management of this chronic condition.

The role of theory in understanding disease management behaviors

Theory provides insight into diverse psychosocial factors that contribute to and maintain health risk behaviors. ${ }^{[10]}$ Theories and their components (i.e., constructs) and processes (i.e., mechanisms or relationships among constructs) can provide insight into human behaviors as related to a variety of influential (or influenced) factors. Not only can we learn the "what," but also the "why" which is guided by empirical work performed across various social and behavioral science disciplines. Women are more likely to engage in a broad range of preventive and health-promoting behaviors than men while men are more likely to engage in over thirty behaviors that have been shown to increase the risk of morbidity, injury, and mortality. ${ }^{[11]}$ At study conducted in 2000 found that men are more likely to engaging in risky behavior, declining to take part in health-promoting activities, and claiming that high-risk behaviors (e.g., alcohol drinking) will not impair performance (e.g., driving) are often demonstrations of the norms of masculinity in the larger culture, and ways in which men construct and reinforce their masculinity. ${ }^{[11]}$

Theories specific to gender may help us understand what contributes to these disparities, and theoreticallydriven systematic inquiry should yield significantly to our understanding of chronic disease self-management. Presumptions about male masculinity and manhood may lead men to either take actions that do harm to them or to refrain from engaging in health-protecting behaviors. Efforts to redefine the cultural meaning of manhood in positive ways will require parallel changes in cultural institutions and social structures to reinforce positive health behaviors in men over the life course. ${ }^{[12]}$ Yet there is insufficient indication that concepts of masculinity or race (or its interactions) are included or accounted for in health behavior research.

Questions about theory often arise when researchers begin to identify research questions that require more complex analysis and need to investigate deeper into their data to explore how illness and health care are conceived and practiced. ${ }^{[13]}$ In particular, what extent have theories and/or constructs specific to race and culture been utilized in diabetes self-care management (SCM) research with African-American men? To address the question, it becomes necessary to examine and determine how well theory and theoretical constructs, particularly those relevant to African-American men, have been applied and adopted in diabetes SCM research.

The research question driving this study seeks to clarify to what extent have theories and/or constructs been utilized in diabetes SCM research with African-American men. Thus, the aim of this paper is to assess the utilization of appropriate (i.e., culturally relevant) theories and/or constructs in diabetes self-management research among African-American men. A secondary aim is to identify factors - specifically barriers-most utilized in research specific to this area. After reviewing type 2 diabetes research literature pertaining to African-American men, research studies with this group have not clearly demonstrated how and why the sustained dietary modifications, blood sugar maintenance, and other self-management customs are not used as consistently as recommended. Therefore, there are not many studies that have analyzed the reasons or barriers for this deficit and or how those living with diabetes view the suggested lifestyle changes.

\section{Materials And Methods}

The current investigation was conducted by first, conducting a systematic exploration of the research literature. This was followed by evaluating each article that meets the inclusionary/exclusionary criteria based on the following parameters utilized by a 2008 study. ${ }^{[14]}$ The analysis for this study adapted the Garrard's matrix method ${ }^{[15]}$ to perform a comprehensive literature review. Therefore, the literature review and analysis helped to frame the methodology that is being used. A systematic search was performed to retrieve peer-reviewed articles addressing SCM among African-American men living with type 2 diabetes. Eight literature databases: Academic Search Complete (EBSCO), ERIC (EBSCO), ScienceDirect (Elsevier), MEDLINE 
(Ovid), Cambridge Scientific Abstracts Databases (CSA), CINAHL, TOPICsearch, and PsycINFO-were searched using keywords such as type 2 diabetes management, selfcare theory, African-American men and type 2 diabetes, and men's health and type 2 diabetes. In addition, all reference sections were purled to ensure the inclusion of any articles omitted during the initial database search for relevant articles. In addition, all reference sections were purled to ensure the inclusion of any articles omitted during the initial database search for relevant articles.

\section{Inclusionary and exclusionary criteria}

Research publications meeting these criteria were included only if the article:

1. Was peer reviewed and published in English between 1996 and 2011,

2. Presented empirical studies (cross-sectional, focus groups, case-control, qualitative, quantitative, longitudinal, group randomized, quasi-experimental, and mixed methods) conducted in the United States, and

3. Investigated type 2 diabetes SCM among AfricanAmerican men.

\section{Theoretically driven empirical articles}

Fifty articles were retrieved and screened for inclusion. Of this number, 49 of these studies were conducted in the United States. An extensive search of the literature databases revealed 31 empirical papers that met the inclusionary and exclusionary criteria. The one research study conducted outside of the United States was automatically excluded. The remainder of the 18 studies conducted in the United States did not address type 2 diabetes SCM among African-American men.

These research articles address other issues pertaining to African-American men such as male masculinity, medical mistrust, perceived body image, health decision-making, discrimination, health information seeking, knowledge of average blood glucose level, depression, fatalism, and fear of having diabetes and its related consequences. In summary, 31 published articles based on research conducted in the United States assessed type 2 diabetes SCM among African-American men.

Results: Scores of the studies and theoretical framework Among the research articles, approximately 12 out of the fifty disclosed a theory and or constructs and how they were utilized. Table 1 shows specifically each scoring category and how many articles coincide with each category. Thirty-four of the fifty articles received a score of "0" (i.e., there was no evidence of theoretical basis driving the research). Two of the fifty articles received a score of " 1 ,"

\begin{tabular}{lcc}
\hline $\begin{array}{l}\text { Table 1: Article scores and usage of theoretical framework } \\
\text { or constructs }\end{array}$ & Score & $\boldsymbol{n}$ \\
\hline Criteria & 3 & 12 \\
\hline Clear identification/operationalization of theory/ & & \\
constructs used & 2 & 2 \\
Use of theory, but inferred (not clearly identified) & 1 & 2 \\
Some evidence of use of theory/constructs & 0 & 34 \\
\hline No evidence of theoretical basis driving the research & & \\
\hline
\end{tabular}

which indicates that the article provided some evidence of theory and use of constructs. Two of the articles received a score of " 2 ," which would have meant that there was use of theory, but that it inferred or not clearly identified.

Lastly, the remaining twelve articles all received a score of "3," which meant that in that article, there was clear identification and operationalization of theory and/or construct use. The constructs used within the reviewed articles were operationalized through a one on one interview, focus group, questionnaire, survey, or predetermined period format. Table 2 shows the breakdown of whether or not a theory or constructs was found in each study. Table 3 below shows the theories, constructs, and models that were found in 12 of the research articles, in which some articles used more than one theory or construct.

\section{DISCUSSION}

After a review of literature, approximately 12 out of fifty articles were found to have clearly stated one or more particular theories within the methodologies section of the research article. Analysis of these publications revealed that most $(n=34)$ were not grounded in a theoretical framework of any kind. The authors of these research articles may have failed to clarify how a theory or theories were applied. While the average score for theory utilization was low (24\%), the result may be due to several reasons such as a limited sample size of African-American men in the research study as compared to African-American women, a lack of trust in research or the researcher, confidentiality, lack of prior participation in research studies, or not being to utilize any particular theory that the researcher(s) felt comfortable in using to address SCM of type 2 diabetes.

Furthermore, the instruments used for analyzing these studies were not piloted or validated. As a result, measurement error is a possibility. Given the scarcity of theory utilization shown from Table 3 clearly translates that there is abundant breach of the "quality" of qualitative research as well as quantitative research. The quality of a research study will be influenced by how the researcher 


\begin{tabular}{|c|c|c|c|c|}
\hline Study & References & Score & Study design & Theoretical framework \\
\hline 1 & Skelley et al. (2008) & 0 & Literature review; intervention protocol & Symptom focused conceptual model \\
\hline 2 & Polzer and Miles (2007) & 3 & Open-ended interviews & Grounded theory \\
\hline 3 & Resnick et al. (1998) & 0 & Secondary data analysis & Not reported \\
\hline 4 & McCleary-Jones (2011) & 3 & Descriptive correlational study & $\begin{array}{l}\text { Health literacy framework; self-efficacy } \\
\text { component of Bandura's social cognitive } \\
\text { theory }\end{array}$ \\
\hline 5 & Rosland et al. (2008) & 0 & Cross-sectional survey & Not reported \\
\hline 6 & Peek et al. (2008) & 1 & Interview focus groups & $\begin{array}{l}\text { Interview guides created based on theory } \\
\text { of planned behavior, ecological model, } \\
\text { and shared decision-making model }\end{array}$ \\
\hline 7 & $\begin{array}{l}\text { Anderson-Loftin and Moneyham } \\
(2000)\end{array}$ & 0 & Focus group & $\begin{array}{l}\text { Nursing care management model (major } \\
\text { concepts: Sick care, health-making, } \\
\text { nurse-client relationship) }\end{array}$ \\
\hline 8 & Hendricks and Hendricks (2000) & 0 & $\begin{array}{l}\text { Diabetes self-management classes; telephone } \\
\text { method for follow-up }\end{array}$ & Not reported \\
\hline 9 & Peek et al. (2011) & 0 & Cross-sectional survey & Not reported \\
\hline 10 & DeWalt et al. (2007) & 0 & Cross-sectional survey & Not reported \\
\hline 11 & Jacobs et al. (2006) & 0 & Focus group & Not reported \\
\hline 12 & Chlebowy et al. (2010) & 0 & Focus group; Chi-square (SPSS) & Not reported \\
\hline 13 & Tang et al. (2008) & 0 & Cross-sectional survey; observational design & Symbolic interaction theory \\
\hline 14 & Sarkar et al. (2006) & 3 & Questionnaire; standardized Cronbach alpha & Self-efficacy theory \\
\hline 15 & Wenzel et al. (2005) & 2 & Focus group & Not reported \\
\hline 16 & Anderson et al. (1996) & 0 & Focus group & Not reported \\
\hline 17 & Liburd et al. (2007) & 0 & In-depth interviews & Not reported \\
\hline 18 & Baptiste-Roberts et al. (2007) & 3 & Cross-sectional & Health belief model \\
\hline 19 & Thompson et al. (2009) & 0 & Focus group & Not reported \\
\hline 20 & Becker et al. (2004) $)^{[18]}$ & 2 & $\begin{array}{l}\text { Based on three large qualitative studies that } \\
\text { examined questions about daily management }\end{array}$ & Not reported \\
\hline 21 & Polzer (2007) & 3 & Structured open-ended interviews & Grounded theory \\
\hline 22 & Duru et al. (2009) & 0 & Case-control study & Not reported \\
\hline 23 & Chesla et al. (2004) & 0 & Questionnaire; semi-structured follow-up interview & Not reported \\
\hline 24 & Bayliss et al. (2003) & 0 & Semi-structured interviews & Not reported \\
\hline 25 & $\begin{array}{l}\text { Holmstrom and Rosenqvist } \\
\text { (2005) }\end{array}$ & 0 & Provider/patient video recordings & Not reported \\
\hline 26 & Carter-Edwards et al. (2004) & 0 & Focus group & Not reported \\
\hline 27 & Onwudiwe et al. (2011) & 0 & Focus group & Not reported \\
\hline 28 & Jones et al. (2008) & 0 & Group sessions & Not reported \\
\hline 29 & Hooker et al. (2012) & 0 & Interviews & Not reported \\
\hline 30 & Batts et al. (2001) & 0 & 2 years study; three intervention visits & Not reported \\
\hline 31 & El-Kebbi et al. (1996) & 0 & Focus group & Not reported \\
\hline 32 & Egede and Bonadonna (2003) & 3 & Focus group & ISAS theory \\
\hline 33 & Hammond and Mattis (2005) & 0 & Survey; Follow-up interviews & Not reported \\
\hline 34 & Aljsem et al. (2001) & 3 & Cross-sectional, correlational study & $\begin{array}{l}\text { Health belief model; self-efficacy } \\
\text { concept }\end{array}$ \\
\hline 35 & Hammond et al. (2010) & 0 & Cross-sectional analysis & Not reported \\
\hline 36 & Hammond (2010) & 1 & Survey & Not reported \\
\hline 37 & Liburd et al. (2007) & 3 & Semi-structured interviews & Not reported \\
\hline 38 & Hammond et al. (2010) & 3 & $\begin{array}{l}\text { Semi-structured interviews with an illness-narrative } \\
\text { framework }\end{array}$ & $\begin{array}{l}\text { Andersen behavioral model; theory } \\
\text { of reasoned action }\end{array}$ \\
\hline 39 & Skelly et al. (2006) & 3 & Semi-structured interviews & Kleinman's exploratory model of illness \\
\hline 40 & Utz et al. (2006) & 0 & Focus group & Not reported \\
\hline 41 & Baptiste-Roberts et al. (2006) & 0 & Cross-sectional analysis & Not reported \\
\hline 42 & Fitzgerald et al. (2000) & 2 & Qualitative analysis & Not reported \\
\hline 43 & Hart et al. (2009) & 0 & Quantitative analysis & Not reported \\
\hline 44 & Heisler et al. (2005) & 0 & Cross-sectional analysis & Not reported \\
\hline 45 & Fisher et al. $(2004)^{[19]}$ & 0 & Cross-sectional analysis & Not reported \\
\hline 46 & Bhattacharya (2012) & 3 & Semi-structured interviews & $\begin{array}{l}\text { Self-determination theory; grounded } \\
\text { theory }\end{array}$ \\
\hline 47 & Anderson-Loftin et al. (2005) & 0 & Longitudinal experimental & Not reported \\
\hline 48 & Walker et al. (2010) & 0 & Quasi-experimental intervention & $\begin{array}{l}\text { Health promotion model; transtheoretical } \\
\text { model }\end{array}$ \\
\hline 49 & Samuel-Hodge et al. (2009) & 0 & Group randomized. Multi-site trial & Not reported \\
\hline 50 & DeCoster and Cummings (2004) & 0 & Exploratory research design & Not reported \\
\hline
\end{tabular}




\begin{tabular}{l} 
Table 3: Theoretical frameworks and constructs that were \\
clearly stated and found in articles \\
\hline Anderson behavioral model \\
Grounded theory \\
Health belief model \\
Health literacy framework \\
Health promotion model \\
Kleinman's exploratory model of illness \\
Nursing care management model \\
Self-determination theory \\
Self-efficacy theory \\
Social cognitive theory \\
Symbolic interaction theory \\
Symptom-focused conceptual model \\
Theory of planned behavior \\
Theory of reasoned action \\
Transtheoretical model
\end{tabular}

attends to theoretical concerns at different stages of the research. Theory can inform qualitative research design and analysis, and theory can also be developed from qualitative analysis. ${ }^{[16]}$ Theoretical considerations play a part at all stages of the research process though this often not made explicit. ${ }^{[13]}$

The findings from this investigation indicate that some diabetes researchers are:

1. Failing to use theory and constructs in directing research;

2. Using theory superficially; or

3. Using theory fully, but failing to be specific and clear about the distribution of the findings.

For these reasons, one could argue that the progress toward improving application and utilization of theory into type 2 diabetes SCM research involving African-American men is still delayed, but is obtaining support in the research literature.

As previously stated earlier in this manuscript, the role that the theoretical framework has in research of any kind is to assist the reader in making logical sense of the relationships of the variables and factors that have been deemed relevant and substantial to the problem at hand. In a sense, theory provides definitions of the relationships between the variables so that the reader can understand the theorized relationships between them. The use of theory makes it possible for researchers to understand, and to translate for policy makers and health care providers, the processes that occur beneath the visible surface and so to develop knowledge of underlying principles. ${ }^{[17]}$ Above all, theory can help people move beyond individual insights gained from their professional lives to a situation where they can understand the wider significance and applicability of the phenomena. ${ }^{[17]}$
Being explicit about the role of theory is part of being transparent to others regarding research design and the analytic process and it is also an important consideration in producing good quality research. ${ }^{[13]}$ The depth and detail of analysis depend upon the focus of the research and available resources, such as time, level of experience and training of the analyst and access to expert advice. The benefit of greater attention to theory in qualitative research is that it enables a more sophisticated approach to the data so that a range of different questions can be asked of the data set. ${ }^{[13]}$

Finally, the application of theoretical frameworks in future research studies would lead researchers to identify the underlying issues associated with how African-American men positively or negatively self-manage type 2 diabetes. While reading and review each article, several major categories of barriers SCM were identified: Shared decisionmaking among African-Americans with diabetes, social support from family and friends, the role of spirituality in SCM, trust and distrust in physicians, masculine role identity factors, and patient perceptions about barriers to SCM. Further research should focus on most common barriers that identified by African-American men living with type 2 diabetes and how those barriers may have a negative impact on SCM. Some barriers, if addressed, may be pliable to interventions that could advance health outcomes.

Financial support and sponsorship

Nil.

Conflicts of interest

There are no conflicts of interest.

\section{REFERENCES}

1. Duru OK, Gerzoff RB, Selby JV, Brown AF, Ackermann RT, Karter AJ, et al. Identifying risk factors for racial disparities in diabetes outcomes: The Translating Research into Action for Diabetes (TRIAD) Study Med Care 2009;47:700.

2. Bayliss EA, Steiner JF, Fernald DH, Crane LA, Main DS. Descriptions of barriers to self-care by persons with comorbid chronic diseases. Ann of Fam Med 2003;1:15-21.

3. Peek ME, Wagner J, Tang H, Baker DC, Chin MH. Self-reported racial/ethnic discrimination in healthcare and diabetes outcomes. Med Care 2011;49:618.

4. Liburd LC, Namageyo-Funa A, Jack L, Gregg E. Views from within and beyond: Illness narratives of African-American men with type 2 diabetes. Diabetes Spect 2004;17:219-24.

5. Hammond WP, Mattis JS. Being a Man About It: Manhood Meaning Among African American Men. Psych of Men \& Mascu 2005;6:114.

6. Aljasem LI, Peyrot M, Wissow L, Rubin RR. The impact of barriers and self-efficacy on self-care behaviors in type 2 diabetes. Diabetes Educ 2001;27:393-404. 
7. Heisler M, Piette JD, Spencer M, Kieffer E, Vijan S. The relationship between knowledge of recent $\mathrm{HbA} 1 \mathrm{c}$ values and diabetes care understanding and self-management. Diabetes Care 2005;28:816-22.

8. Samuel-Hodge CD, Keyserling TC, Park S, Johnston LF, Gizlice Z, Bangdiwala SI. A randomized trial of a church-based diabetes self-management program for African Americans with type 2 diabetes. The Diabetes Educ 2009;35:439-54.

9. DeCoster VA, Cummings S. Coping with type 2 diabetes: Do race and gender matter?. Soc Work in Health Care 2005;40:37-53.

10. Jones RA, Utz SW, Williams IC, Hinton I, Alexander G, Moore C, et al. Family interactions among African Americans diagnosed with type 2 diabetes. Diabetes Educ 2008;34:318-26.

11. Bhattacharya G. Psychosocial impacts of type 2 diabetes self-management in a rural African-American population. $\mathrm{J}$ of Immigrant and Minority Health 2012;14:1071-81.

12. Fisher L, Laurencin G, Chesla CA, Skaff MM, Mullan JT, Gardiner PS, et al. Depressive affect among four ethnic groups of male patients with type 2 diabetes. Diabetes Spectrum 2004; 17:215-9.

13. Hammond WP, Matthews D, Corbie-Smith G. Psychosocial factors associated with routine health examination scheduling and receipt among African American men. J National Medical Association 2010;102:276-89.

14. Anderson-Loftin W, Moneyham L. Long-term disease management needs of southern African Americans with diabetes. Diabetes Educ 2000;26:821-32.

15. Anderson-Loftin W, Barnett S, Bunn P, Sullivan P, Hussey J, Tavakoli A. Soul food light culturally competent diabetes education. Diabetes Educ 2005;31:555-63.

16. Sarkar U, Fisher L, Schillinger D. Is self-efficacy associated with diabetes self-management across race/ethnicity and health literacy? Diabetes Care 2006;29:823-9.

17. Walker EA, Stevens KA, Persaud S. Promoting diabetes self-management among African Americans: An educational intervention. $\mathrm{J}$ of Health Care for the Poor and Underserved 2010;21:169-86.

18. Becker G, Gates RJ, Newsom E. Self-care among chronically ill African Americans: Culture, health disparities, and health insurance status. American J of Public Health 2004;94:2066-73.

19. Fisher L, Skaff MM, Chesla CA, Chun KM, Mullan JT, Kanter RA, Gardiner PS. Disease management advice provided to AfricanAmerican and Chinese-American patients with type 2 diabetes. Diabetes Care 2004;27:2249-50. 